\title{
Moving Beyond Weak Identifiers for Proxemic Interaction
}

\author{
Henrik Sørensen and Jesper Kjeldskov \\ Department of Computer Science / Research Centre for Socio-Interactive Design \\ Aalborg University, Denmark \\ \{hesor, jesper\}@cs.aau.dk
}

\begin{abstract}
Identification of people and their position is essential in the design of proxemic interactions. The smartphone often plays an important role in positioning systems, due to its mobility, computational power and sensory capabilities. Studies however show that perceived proximity to our phone is significantly closer than what is actually the case. This makes the smartphone a weak identifier for applications that need to track persons. With a focus on feasible interaction design, we present a concept and prototype of a platform, which seek to support proxemic interaction beyond weak identifiers. The concept is a lightweight, low-cost, platform, offering a high update-rate that in particular focuses on support for the identity, location and distance dimensions of proxemic interaction. An evaluation of the platform validates its potential use for proxemic interactions but also reveals challenges and limitations that need to be addressed.
\end{abstract}

\section{Categories and Subject Descriptors \\ H.4.m [Information Systems Applications]: Miscellaneous}

\section{General Terms}

Experimentation, Human Factors.

\section{Keywords}

Proxemic interaction, weak identifiers, domestic environment

\section{INTRODUCTION}

Greenberg et al. operationalize proximity within ubiquitous computing (ubicomp) into five dimensions: Distance, orientation, movement, identity, and location [4]. The ability to position and track people around is essential in the design of proxemic interactions. It therefore seems reasonable to make a connection between the work in indoor positioning and proxemic interaction. Previous work on indoor positioning systems has primarily focused on improvement of accuracy and making the infrastructure and algorithms robust to changes in the environment. The implications of solutions to practical usefulness, have however not been considered to the same degree. Achieving a high quality positioning and tracking is of course imperative to the general usefulness of the technology, but there are other factors, which influence how appropriate a positioning system is to applications within proxemic interaction.

Permission to make digital or hard copies of all or part of this work for personal or classroom use is granted without fee provided that copies are not made or distributed for profit or commercial advantage and that copies bear this notice and the full citation on the first page. To copy otherwise, or republish, to post on servers or to redistribute to lists, requires prior specific permission and/or a fee.

MoMM2013, 2-4 December, 2013, Vienna, Austria.

Copyright 2013 ACM 978-1-4503-2106-8/13/12 ...\$15.00.
In the research area of ubicomp, it is often assumed that smartphones are representative indicators for the position of people. Especially in the work of indoor positioning, solutions are often dependent on smartphones in both infrastructure and application. The work of Patel et al. [10] and Dey et al. [2] however reveal an issue with the use of phones as positioning devices. Contrary to common perception, we do not carry our mobile phone around most of the time. This makes the phone unsuitable, as a user representation, in cases where it is the user and not the device we are trying to keep track of. Another issue with the use of smartphones as identifiers is missing the proxemic relationship between the phone and the person using it.

The distance between individual and smartphone is a significant noise factor on top of a positioning algorithm, where effort is put into optimization of performance. It is in other words not very useful to have obtained accuracies down to a few centimeters, if the distance between user and phone is several meters. The contribution of this paper is to present a concept, which facilitates strong identifiers for a clear distinction between tracking people and tracking devices. The focus is on practical feasibility of interacting designs facilitating the identity, location and distance dimensions of proxemic interactions spanning multiple rooms.

\section{RELATED WORK}

\subsection{Proxemic Interaction}

When we talk about proxemic interaction, it refers to the work of Greenberg et al. on operationalizing the concept of proximity within ubicomp and developing a framework for proxemic interactions [4]. They extend Hall's notion of proxemics [5] to consider distance, orientation, movement, identity and location as dimensions of spatial relationships between people, devices, and non-digital things. In their work, they have developed a proximity toolkit for fast prototyping of proxemic interactions [9]. Their focus is on the complete ecology of small space ubicomp environments, meaning that they have made a great contribution to the fine-grained interaction in room-sized environments. What they have not explored are systems in larger spaces, like a house/apartment, where interaction spans across rooms. Although there is no theoretical scalability issue with their prototyping framework and toolkit, it relies on expensive equipment deployed in every area relevant to the application. The toolkit supports different types of sensory input, through a plugin mechanism, like the Microsoft Kinect as a cheaper alternative to the Vicon motion capture system based approach.

\subsection{Indoor Positioning}

Identification of persons and their position is an important aspect of proxemic interactions. The notion of proxemic interaction therefore has a certain relation to the area of indoor positioning. The approach seen in the Proxemic Toolkit [9] is for instance based on what can be referred to as scene analysis in indoor positioning [12]. Quite a large body of work has previously addressed indoor positioning suitable to cover multi-room indoor 
environments. In work on both infrastructure for, and application of indoor positioning, the smartphone often plays an important role. Indoor positioning systems based on radio frequency (like WLAN or Bluetooth) are the most common in literature [12]. In this case, smartphones are often used as the tracking device [1] and/or as providers of data for a radio map [6]. Other cases utilize various inertial sensors from the smartphone, like the accelerometer or compass [7], and sometimes the smartphone is a subpart of a larger sensor network [11]. It is however not only in the direct work of indoor positioning the phone plays an important role. Activity recognition is an example that uses data from smartphones to infer human activity [8].

\subsection{The Smartphone as a Weak Identifier}

Patel et al. questioned the assumption that we carry our phone around at all times in their paper from 2006 [10], where they point out issues in real world contexts. Through an empirical study, they found that their participants only had their phones turned on and within arm's reach (1-2 meters) on an average of 50\% of the time. They furthermore found that participants were more likely to keep the phones outside arm's reach when at home, compared to when they were away from home.

Dey et al. followed up on the work of Patel et al. in a paper from 2011 [2], by replicating the experiment in a time where the smartphone had made its impact. The initial hypothesis was that smartphone users would carry their phones around more than the previous generation of mobile phone users. Their results were however very similar to that of Patel et al. and revealed that the phone was still turned on and within arm's reach of the owner only $53 \%$ of the time.

What the two studies, on the proximity of mobile phones, have shown is that the position of the phone is not always equal to that of the user, even in a smartphone era where we constantly use them in various contexts. In the interaction design of proxemic interactions, we therefore have to be careful about the roles imposed on the smartphone and not see it as a necessary part of the solution just because it is convenient.

\section{CONCEPT}

Our concept is inspired by systems like the ActiveBadge by Want et al. [13] where wearable tokens are used to infer user position in an indoor environment. The ActiveBadge is an example of Weiser's notion of tabs as the smallest component of virtual embodiment in his vision of ubiquitous computing [14]. The goal of our concept is to provide a lightweight, low-cost platform for proxemic interactions spanning across rooms in indoor locations. The focus is on the identity, location, and distance of persons and devices and to provide an architecture independent of versatile devices like smartphones as personal identifiers.

The platform is based on communication between two component types: Signal transmitters, and measuring units. Figure 1 illustrates the concept where measuring units, strategically placed in the environment, continuously approximate the position of small uniquely identifiable signal transmitters. The idea is to allow for a flexible platform, which can be set up in an ad-hoc manner. One of the measuring units acts as a position server and continuously keeps track of each signal transmitter's position. The position server is furthermore responsible for facilitating information on proxemic relationships to relevant applications. The concept emphasizes the power of small, specialized tokens as an alternative to the smartphone on which a multitude of functionality is already imposed. As the smartphone evolves, so does the variety of applications of it. It is however not always convenient to have functionality centralized in a single device and there is a certain power in simple devices with simple purposes.

Implementing an effective positioning algorithm is outside the scope of this paper. Instead, we address specific issues necessary to handle before we can benefit from ongoing work on highperformance positioning and use it in the design of proxemic interactions.

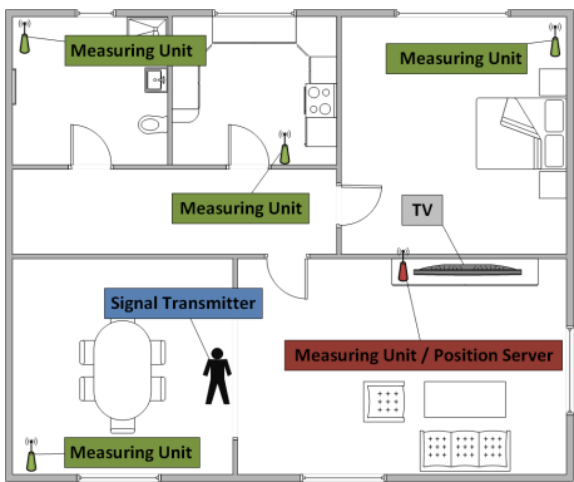

Figure 1. Floor plan illustrating an example setup.

\subsection{Independence of Smartphone}

A crucial criterion for our concept is to be independent of the use of smartphones. This is primarily achieved by supporting what can be referred to as remote positioning [3]. In remote positioning, the tracked device takes on the role as a signal transmitter. A number of stationary measuring units with known locations receive the signal. Because the measuring units carry the primary responsibility and computational workload, the requirements of the tracked device are low. This allows for flexibility in form, size and energy consumption.

\subsection{Support for Proxemic Interaction}

There are several approaches to indoor positioning. Common to the different techniques is the measurement of some physical quantity that changes according to the position of the tracked item. Torres-Solis et al. identifies radio frequency, photonic, sonic, and inertial as the dominant technologies in the literature [12]. Any positioning system inherently supports the location dimension and to some degree distance. An advantage of wireless positioning is that the used technologies provide a unique identifier of the tracked device, namely a hardware address. Image analysis of camera streams, like in the Microsoft Kinect or Vicon systems, makes it more difficult to support the identity aspect.

\subsection{Dedicated Signal Transmitters}

By utilizing wearable dedicated signal transmitters, the smartphone is treated similarly to other electronic devices, instead of a representation of the user. Separating the signal transmitters from the smartphone, does not only allow for a closer tracking of the individual. The concept of having small devices dedicated to provide proxemic input enables other interesting opportunities as well. One is adding signal transmitters to otherwise non-electronic objects in the home, like furniture. The other is the development of specialized tokens serving as tangible interfaces.

\subsection{Low Cost}

Regarding cost in terms of money, we aim at a setup that is flexible and makes it possible to do simple stuff without the need for high-grade professional equipment. Work in wireless indoor 
positioning often builds on top of a network of wireless access points. The systems typically presented, are considered very lowcost as the hardware infrastructure is already present. In smaller spaces like private homes, we can however not assume that an infrastructure with multiple $\mathrm{Wi}-\mathrm{Fi}$ access points for triangulation is available. We therefore find it important to keep the cost down without relying too much on existing infrastructure.

Another cost aspect is the energy consumption of the signal transmitter. Even though the form factor is a wearable device, with the potential of being carried within close proximity, it still needs to be left behind while charging. It is therefore important to have a device with low energy consumption as such small devices rarely have a high-capacity power source. Having specialized devices partly achieve that, which unlike a smartphone does not need to operate power consuming hardware like a high-resolution screen, multi-core CPU and several network-interfaces.

\subsection{High Update Rate}

A very high level of accuracy is not very useful if it takes 10 seconds to obtain it. The proxemic interactions become powerful through an environment that can react to the current relations between people, devices and objects and not the relations as they were a while ago. Although our architecture has an overhead in relaying measurements to the position server, the bottleneck is often the time it takes to collect measurements. It is therefore important that the signal transmitters are continuously available and that the measuring units and the server can handle measurements at a high rate from multiple sources simultaneously.

\section{PROTOTYPE}

The goal of the prototype is to realize a proof-of-concept implementation of the presented concept. It relies on low-cost ofthe-shelf hardware components and a number of software components. The following describes the hardware setup as well as important aspects of the software implementation.

\subsection{Hardware}

The Raspberry Pi Model B constitutes the hardware platform for both measuring units and the central server. It is a single-board computer featuring a $700 \mathrm{MHz}$ ARM11 CPU, 512MB RAM, Broadcom VideoCore IV GPU, HDMI output and $2 \times$ USB 2.0 ports, meaning that it is capable of both hosting the proxemic interaction platform and to some extent applications facilitating the platform. An advantage of the Raspberry $\mathrm{Pi}$ is its price ( $35 \$$ without peripherals) and that it is powered by a $5 \mathrm{~V}$ micro USB adapter (standard smartphone charger) limiting power usage. The Raspberry Pi uses an SD-card as the main storage, making it easy to clone for deployment on several units. A Bluegiga BLED112 class 2 USB adapter enables Bluetooth Low Energy (LE) capabilities. It contains its own Bluetooth stack accessed through a developer API. Connection to a wireless network is through a Wireless LAN USB adapter.

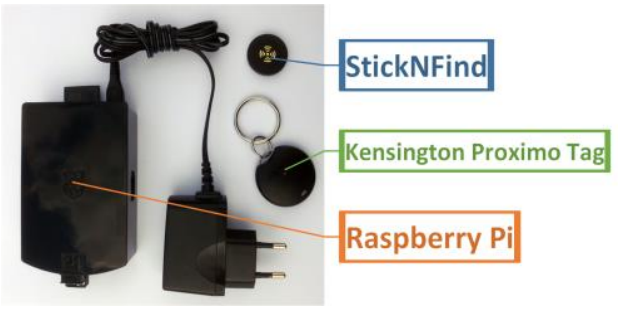

Figure 2. Hardware components used in the platform.
The signal transmitters used are Kensington Proximo and StickNFind Bluetooth LE tags, both powered by a standard button cell battery. Because of the implementation of the Bluetooth low energy profile, they have the potential to run on a single battery for up to half a year. In principle, any Bluetooth LE device that advertises its presence can be tracked, including Bluetooth LE capable smartphones, tablets, etc. The different hardware components are shown in Figure 2.

\subsection{Architecture}

The platform consists of the three types of components as presented in the concept description: A number of signal transmitters, a number of measuring units and a central server. The Raspberry Pi units used for the measuring units and the central server runs a Debian-based Linux distribution (Raspbian) optimized for the Raspberry Pi. The software components are however developed in Java and runs on a virtual machine.

As illustrated in Figure 3, each measuring unit hosts an instance of a developed proxemic client application responsible for retrieving measurements from the signal transmitters, preparing a message and send it to the message broker. Messages can be retrieved either by the server application or directly by proxemic applications that need information about a specific measuring unit's distance to signal transmitters.

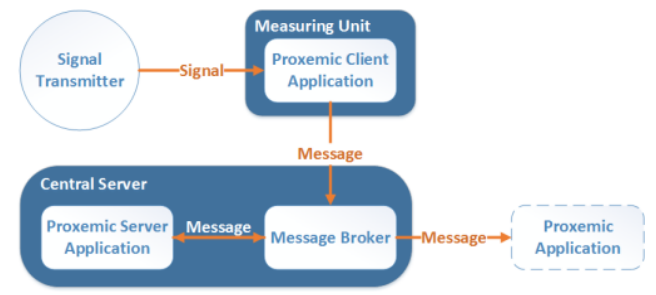

Figure 3. Overall architecture of the proxemic platform.

The proxemic server receives messages from clients through the message broker, which it uses to maintain the position of each signal transmitter. The central server can additionally host an instance of the proxemic client application, making it capable of retrieving measurements from signal transmitters itself (not included in Figure 3 for simplicity). Whenever a change in the position of a signal transmitter is determined, the server sends a special message to the message broker, which any listening proxemic applications retrieves. In our prototype, Multicast DNS is used to resolve hostnames through the Zeroconf implementation Avahi. It allows the proxemic client application to refer to the server as "server.local" instead of a specific IP address. Clients can therefore resolve the address of the server and start passing messages without prior configuration to a specific network (other than connecting to a wireless LAN).

\subsection{Proxemic Measurements}

In our case, Bluetooth LE is the technology used to perform measurements usable to proxemic interactions. The attractive quality of Bluetooth LE is the potential for a fast update rate and low power footprint. We exploit the advertiser and scanner roles, in the Bluetooth LE specification, normally used to discover nearby devices. These procedures are the Bluetooth LE equivalent to traditional Bluetooth inquiry procedures, similarly used in Bluetooth-based indoor positioning. Each Bluetooth LE tag acts as an advertiser and uses an advertising procedure to make itself discoverable by nearby devices. The advertising procedure performs a unidirectional broadcast at a fixed interval, which nearby scanning devices can receive. The interval, in which 
broadcasts occur, is dependent on the specific implementation of the advertising procedure.

When the proxemic client application starts, it resolves the server address and creates a connection to the message broker. It then connects to the Bluetooth software stack and initiates a scanning procedure. The scanning procedure listens for broadcasts and gets an estimate of the distance to the advertiser by measuring the signal strength. The Bluetooth LE adapter returns the signal strength as an RSSI value measured in $\mathrm{dBm}$. The used adapter returns RSSI values in the range from -103 to $-38 \mathrm{dBm}$.

When a broadcast is received a message is constructed which is passed to the message broker. The values passed to the server are a measurement id, the client id, the MAC address of the Bluetooth tag, and the measured RSSI. The proxemic server application receives the messages via the message broker and can centrally analyze the proxemic relations. The implemented algorithm simply keeps track of discovered signal transmitters, estimated distance to measuring units, and which measuring unit they are within closest proximity of. Whenever a change happens for any of the discovered signal transmitters, the server application publishes a message to the message broker, which can be received by applications that need to react to it.

\subsection{Machine-to-Machine Communication}

The platform architecture relies on a central proxemic server. It is therefore not only important to be able to collect RSSI measurements at a high rate, but furthermore to enable fast machine-to-machine (M2M) communication. Our platform makes use of a publish/subscribe pattern and in particular an implementation of the lightweight Message Queuing Telemetry Transport (MQTT) protocol.

A message broker maintains a number of topics and is responsible for relaying messages from publishing clients to subscribing clients of particular topics. In our architecture, the MQTT broker resides on the central server. Topics are organized in a simple hierarchy illustrated in Figure 4. Each client application publishes messages containing RSSI updates to a subtopic in the format "rssi/[CLIENT_ID]". The server application subscribes to the toplevel topic "rssi/\#". The \# wildcard defines a subscription to the topic and all of its subtopics, meaning it will receive RSSI updates from all connected clients. If an application needs to keep track of tags' distance to a specific client, it can simply subscribe to the relevant subtopic. Alternatively applications can subscribe to a dedicated top-level topic "location_change/" which is updated by the server whenever any tag changes location. A usable feature of the MQTT protocol is that topics are created dynamically as messages are published to it. It is therefore not necessary to keep track of connected clients on either the broker or the server.

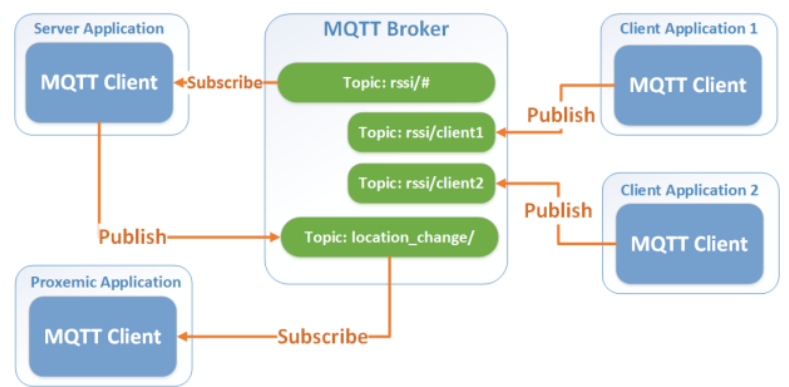

Figure 4. Machine-to-machine communication through MQTT in an example setup with two connected client applications.

\section{Performance Evaluation}

Performance evaluations were conducted to validate the developed platform. The first evaluation served two purposes: To evaluate the update rate of the implemented proxemic platform as an integrated system and to evaluate observed relation between RSSI measurements and distance. We placed a measuring unit and a Kensington Proximo Tag in one room and the central server, and a customer-grade wireless router in a room next to it. Measurements were collected over a 2-minute period at a distance of $0,1,2,3,4$ and 5 meters respectively. The server was responsible for logging timestamps, as messages arrived, in order to evaluate the prototype setup as an integrated system.

We present the results of the performance evaluation descriptively as our intention is not to provide statistically founded insights into radio frequency based indoor positioning. Our intention is to provide a performance overview of the prototype, in order to validate its potential as a cheap and responsive platform for proxemic interaction applications independent of smartphones. The evaluation shows the system's capability to update the distance on the server from one tag to a measuring unit between 76 and 95 times over a duration of $2 \mathrm{~min}$. The result is a mean delay from 1.26 to 1.59 seconds between updates. We did not find any correlation between the delay and the distance. The distance similarly did not seem to influence the standard deviation of the delays. Instead, we observed that updates were distributed evenly over the $2 \mathrm{~min}$. period regardless of distance.

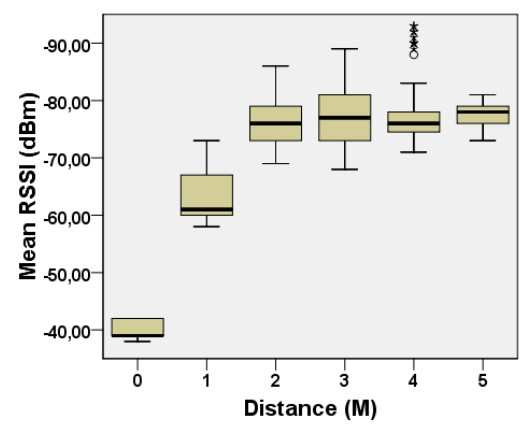

Figure 5. RSSI measurements for each distance.

Figure 5 shows the mean RSSI values measured as a function of distance and reveals a desired decrease in signal strength as the distance increases. It is however clear that there is no linear relationship between the two factors and the curve already starts to level off after 2 meters. The evaluation furthermore showed that when distance increased beyond 5 meters, measurements either would be very similar to measurements from 5 meters or would return invalid values. A closer look at the data reveals another challenge. An overlap is evident in the distribution of measurements even at low distances. It will therefore be challenging to obtain a close estimate of distance based on few measurements. If measurements are not stable, the benefit of a high update-rate can therefore be lost.

We performed an additional evaluation to validate the stability of the prototype over two 12-hour trials in a private household, where the two participants wore a Bluetooth LE tag. The evaluation have shown the potential of the prototype as a flexible way of collecting information used to facilitate proxemic interactions. Because neither measuring units nor the server needs to connect to new signal transmitters, they can easily move in and out of the range in an ad-hoc manner without influencing the overall system. 


\section{Discussion}

\subsection{Proxemic Interaction}

An important aspect of proxemic interaction within ubicomp environments is to consider more dimensions than distance. It is clear that a lot can be learned from the large body of work on indoor positioning, to create means to provide proxemic information on other dimensions. The use of small, low-power wearable tags furthermore constitutes stronger personal identifiers, which opens up for different novel proxemic interactions where the proxemic relationship between user, smartphone, and other devices can be meaningful.

Having wearable sensors as a part of a context aware environment is of course not a novel contribution in our work. As mentioned, early attempts, as the ActiveBadge by Want et al. [13], has greatly inspired out work. We do however think it is valuable to rethink the opportunities such specialized devices provide in relation to proxemics interactions. In terms of form of wearable signal transmitters, emerging smart watches could be an interesting alternative to the use of the smartphone as a proxemic sensor. The primary intended use of smart watches is as an accessory to the smartphone. It would however be interesting to see how it could work as a signal transmitter for a platform, like proposed in this paper, to create a personalized experience.

\subsection{Robustness of Indoor Positioning}

The implementation of a sophisticated indoor positioning system is out of the scope of this paper. The platform does however imply the need for an implementation of a positioning algorithm on top of the radio frequency measurements of the Bluetooth LE devices. What our performance evaluation have shown is definitely a need for something more than comparing RSSI values for reliable location estimates. Low-resolution distance estimates to individual measuring units, and comparison of closest measuring unit in strategic setups is however achievable in its current state.

The observed non-linear behavior seen in the results of the performance evaluation seems compliant with the empirically based log-distance path loss model of indoor radio propagation. The alternative frequency hopping of Bluetooth LE compared to traditional Bluetooth furthermore enables a much faster update rate. It does however seem like the compact design of Bluetooth LE tags makes the antenna very sensitive to external influences. Putting the tag inside a pocket, instead of wearing it on the outside of clothing, can for instance quite significantly influence measurements. We would however like to stress the role of the platform as a lightweight alternative to for example the Proximity Toolkit [9] in situations where a system is deployed in multi-room locations and where the identity of the tracked persons and responsiveness is more important than micro-level proxemic relations. Although a high update-rate has been important to our concept, this is seen in comparison to radio frequency based indoor positioning, rather than that of the motion capture systems used in the Proximity Toolkit.

\section{Conclusion}

We have presented a lightweight, low-cost platform, which offers the means to create proxemic interactions independent of weak identifiers. The flexibility of both architecture configurations and various types of signal transmitters, in term of size and form, does however come with a challenge in obtaining robust measurements usable in fine-grained distance and location estimates. We do however think it is important also to consider other aspects in the development of indoor positioning systems and proxemic interactions than accuracy and precision of measured values. Our approach has focused on a closer tracking through wearable Bluetooth LE tags, which at the same time offers a low power usage, and high update rate compared to similar work in radio frequency based indoor positioning.

\section{REFERENCES}

[1] F. Aloul, A. Sagahyroon, A. Al-Shami, I. Al-Midfa, and R. Moutassem. 2009. Using mobiles for on campus location tracking. In Proc. MoMM 2009. ACM (2009), 231-235.

[2] Dey, A.K., Wac, K., Ferreira, D., Tassini, K., Hong, J.-H., and Ramos, J. Getting closer: an empirical investigation of the proximity of user to their smart phones. In Proc. UbiComp 2011. ACM (2011), 163-172.

[3] Drane, C., Macnaughtan, M., and Scott, C. Positioning GSM telephones. Communications Magazine, IEEE 36, 4 (1998), 46-54, 59.

[4] Greenberg, S., Marquardt, N., Ballendat, T., Diaz-Marino, R., and Wang, M. Proxemic interactions: the new ubicomp? interactions 18, 1 (2011), 42-50.

[5] Hall, E.T. The Hidden Dimension. Doubleday (1966).

[6] Jiang, Y., Pan, X., Li, K., Lv, Q., Dick, R.P., Hannigan, M., Shang, L. ARIEL: automatic wi-fi based room fingerprinting for indoor localization. In Proc. UbiComp 2012. ACM (2012), 441-450.

[7] Kjærgaard, M.B., Wirz, M., Roggen, D., and Tröster, G. Detecting pedestrian flocks by fusion of multi-modal sensors in mobile phones. In Proc. UbiComp 2012. ACM (2012), 240-249.

[8] Lau, S.L., König, I., David, K., Parandian, B., Carius-Düssel, C., and Schultz, M. Supporting patient monitoring using activity recognition with a smartphone. In Proc. ISWCS 2010, IEEE (2010), 810-814.

[9] Marquardt, N., Diaz-Marino, R., Boring, S., and Greenberg, $\mathrm{S}$. The proximity toolkit: prototyping proxemic interactions in ubiquitous computing ecologies. In Proc. UIST 2011. ACM (2011), 315-326.

[10] Patel, S.N., Kientz, J.A., Hayes, G.R., Bhat, S., and Abowd, G.D. Farther than you may think: an empirical investigation of the proximity of users to their mobile phones. In Proc. UbiComp 2006. Springer-Verlag (2006), 123-140.

[11] Teixeira, T., Jung, D., and Savvides, A. Tasking networked CCTV cameras and mobile phones to identify and localize multiple people. In Proc. UbiComp 2010. ACM (2010), 213-222.

[12] Torres-solis, J., Falk, T.H., and Chau, T. A review of indoor localization technologies : towards navigational assistance for topographical disorientation. Ambient Intelligence, InTech (2010), 51-84.

[13] Want, R., Hopper, A., Falcão, V., Gibbons, J. The active badge location system. ACM Transactions on Information Systems (TOIS), 10, 1 (1992), 91-102

[14] Weiser, M. The computer for the 21st century. SIGMOBILE Mob. Comput. Commun. Rev. 3 , 3 (1991), 3-11. 